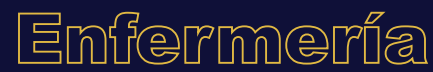

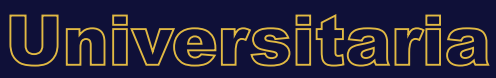

\section{La técnica Delphi para el rediseño y validación de un instrumento que mide la comunicación enfermera-paciente}

\author{
Using the Delphi method to redesign \\ and validate an instrument measuring \\ nurse-patient communication
}

\begin{abstract}
A técnica Delphi para o redesenho e validação de um instrumento que mede a comunicação enfermeira-paciente
\end{abstract}

S. Hernández-Corral ${ }^{\mathrm{at}^{*}}$, M.C. Müggenburg-Rodríguez Vigil ${ }^{\mathrm{b}}$, S.S. Olvera-Arreolac ${ }^{c_{3}}$

ORCID:

${ }^{\mathrm{a}} \mathrm{0} 000-0003-4292-2380$

b0000-0002-0497-8685

c00oo-0oo2-6027-6573

'Subdirección de Enfermería, Instituto Nacional de Rehabilitación

Luis Guillermo Ibarra Ibarra, Ciudad de México, México

${ }^{2}$ Coordinación de Investigación, Escuela Nacional de Enfermería y Obstetricia,

Universidad Nacional Autónoma de México, Ciudad de México, México

${ }^{3}$ Dirección de Enfermería, Instituto Nacional de Cardiología,

Ignacio Chávez, Ciudad de México, México

Recibido: 20 febrero 2019/Aceptado: 14 agosto 2019

RESUMEN

Introducción: La evaluación de la comunicación entre la enfermera y el paciente da cuenta de la dimensión interpersonal en la calidad del cuidado. Esta relación ha sido estudiada desde diversos enfoques con énfasis en algunos aspectos, como el diseño y validación de instrumentos.

*Autor para correspondencia. Correo electrónico: shcorral@gmail.com https://doi.org/10.22201/eneo.23958421e.2019.4.596

1665-7063/@ 2019 Universidad Nacional Autónoma de México, Escuela Nacional de Enfermería y Obstetricia. Este es un artículo Open Access bajo la licencia CC BY-NC-ND (http://creativecommons.org/licenses/by-nc-nd/4.o/). 
Objetivo: Rediseño y validación de contenido de un instrumento que mide comunicación enfermera-paciente, con base en la técnica Delphi.

Metodología: Se enfoca al rediseño y validación de un instrumento, con la aplicación de la técnica Delphi, con la participación de enfermeras expertas, sensibles a la relación terapéutica; se realizaron tres rondas con integración de contenidos por las coordinadoras y la aplicación de los coeficientes de validación de contenido por reactivo (CVR) y validación de contenido integral (CVI).

Resultados: El CVI del instrumento en cuestión (CECOP-23) en la primera ronda fue 0.84, posterior a ello se eliminaron cuatro reactivos y se sustituyeron dos que fueron sugeridos por los expertos. En la segunda ronda el instrumento alcanzó un CVI de 0.95, el grupo sugirió la utilización de términos más sencillos que propiciaran la cercanía con el paciente. En la tercera ronda se obtuvo un CVI de o.96. El CECOP quedó integrado por 21 reactivos, diez que exploran empatía y once que exploran respeto.

Conclusiones: El trabajo con los expertos permitió analizar los reactivos del CECOP-23, obteniendo el CECOP-21, con reactivos de mayor calidez y exploración más precisa, cumpliendo con el objetivo de diseñar un instrumento que explore ambas dimensiones manteniendo el equilibrio entre la empatía y el respeto.

Palabras clave: Comunicación enfermera-paciente; respeto; empatía; México.

\begin{abstract}
Introduction: The assessment of the communication between nurses and patients is part of the interpersonal dimension of quality of care. This relationship has been studied from diverse points of view which focus on issues such as the design and validation of measuring instruments.

Objective: Using the Delphi method, to redesign and validate a nurse-patient communication measuring instrument.

Methodology: Together with expert and sensitive to the therapeutic relationship nurses, the Delphi method was used to redesign and validate a communication measuring instrument. The coordinators assessed the contents in three cycles calculating the Item Content Validity and Integral Content Validity coefficients.

Results: The Integral Content Validity of the CECOP-23 instrument was 0.84 in the first cycle. Four items were deleted and two were substituted in agreement with the suggestions of the experts. The Integral Content Validity of the instrument was 0.95 in the second cycle. The group suggested using simpler terms which could foster closeness to the patient. The Integral Content Validity was 0.96 in the third cycle. The CECOP instrument was finally constituted by 21 items, 10 exploring empathy and 11 exploring respect.

Conclusions: Working along with the experts prompted the analysis of the CECOP-23 instrument in order to produce the CECOP-21 which has more precise and "warm" items. The objective of redesigning and instrument which, maintaining the equilibrium between empathy and respect, could measure the nurse-patient communication was achieved.
\end{abstract}

Keywords: Nurse-patient communication; respect; empathy; Mexico.

\title{
RESUMO
}

Introdução: A avaliação da comunicação entre a enfermeira e o paciente dá conta da dimensão interpessoal na qualidade do cuidado. Esta relação tem sido estudada desde diversos enfoques com ênfase em alguns aspectos, como o desenho e a validação de instrumentos. 
Objetivo: redesenho e validação de conteúdo de um instrumento que meça a comunicação enfermeira-paciente, com base na técnica Delphi.

Metodologia: Foca-se no redesenho e validação de um instrumento, com a aplicação da técnica Delphi, com a participação de enfermeiras experientes, sensíveis à relação terapêutica; realizaram-se três ciclos com integração de conteúdos pelas coordenadoras e a aplicação dos coeficientes de validação de conteúdo por item (CVR) e a validação de conteúdo integral (CVI). Resultados: O CVI do instrumento em questão (CECOP-23) na primeira volta foi o.84, posterior a isto, eliminaram-se quatro itens e substituíram-se dois que foram sugeridos pelos especialistas. Na segunda volta o instrumento atingiu um CVI de 0.95, o grupo sugeriu a utilização de termos mais simples que propiciaram a proximidade com o paciente. Na terceira volta obteve-se um CVI de 0.96. O CECOP ficou integrado por 21 itens, dez que exploram empatia e onze respeito. Conclusões: O trabalho com os especialistas permitiu analisar os itens de CECOP-23, obtendo o CECOP-21, com itens de maior qualidade e exploração mais precisa, cumprindo com o objetivo de desenhar um instrumento que explore ambas as dimensões mantendo o equilíbrio entre a empatia e o respeito.

Palavras chave: Comunicação enfermeira-paciente; respeito; empatia; México.

\section{INTRODUCCIÓN}

La importancia que tiene la comunicación enfermera-paciente en la oferta de servicios personalizados para mantener y desarrollar la salud y el bienestar de las personas motiva al estudio de este fenómeno en México․ La trascendencia del tema ha originado que se analicen diversos aspectos: los participantes de la interacción, la experiencia profesional y la sensibilización humanística de las enfermeras, las técnicas empleadas en los entrenamientos para la comunicación, la duración de los cursos y el efecto de programas en habilidades sociales sobre los pacientes como elementos fundamentales para el establecimiento de estrategias que coadyuven al mejoramiento de la calidad de los servicios hospitalarios ${ }^{2-4}$.

Con base en la limitante enunciada en un artículo que aborda el diseño y validación de un instrumento que mide comunicación enfermera paciente en población mexicana ${ }^{5} \mathrm{y}$ el interés de los autores en profundizar en la exploración de comportamientos de empatía y respeto que facilitan la comunicación terapéutica ${ }^{6}$, se decide retomar el instrumento que mide el Comportamiento de Enfermeras en Comunicación Observado por los Pacientes (CECOP-23) y someterlo a juicio de expertos con la finalidad de rediseñar y validar este instrumento. Cabe mencionar que se tiene previsto realizar validación de contenido por expertos mediante la aplicación de la técnica Delphi.

Para contextualizar el desarrollo del tema se buscó en la literatura quiénes y cómo han medido esta relación interpersonal7 la mayoría de los instrumentos se han aplicado a pacientes hospitalizados, algunos en consulta externa, durante su etapa de hospitalización y ocasionalmente después del egreso.

Las mediciones en la mayoría de los casos se abocaron a comunicación efectiva, habilidades sociales, empatía, trato digno y/o escucha activa ${ }^{8-10}$, fueron medidas con escalas tipo Likert; el propósito de las mediciones se centró principalmente en la evaluación de la comunicación entre la enfermera y el paciente y en su repercusión en la dimensión interpersonal de la calidad del cuidado. Se reconocieron algunas oportunidades de mejora como: permanecer más tiempo cerca del paciente y optimizar las habilidades de comunicación interpersonal. 
Los mecanismos de validación de los instrumentos empleados fueron validez de contenido por expertos y/o validez de constructo. En el primer caso algunos autores se apoyaron con la aplicación de la técnica Delphi, esta última ofrece alcanzar un consenso fiable entre las opiniones de un grupo de expertos; ya que consiste en un trabajo grupal a distancia, en la que los integrantes no tienen una comunicación directa a lo largo de varias rondas ${ }^{11}$. En cada nueva ronda, los expertos deben evaluar los mismos elementos (tienen posibilidad de cambiar sus respuestas o comentarios), pero cuentan con información de las opiniones que el resto de expertos ofreció en la ronda anterior, de modo que las valoraciones muestran una tendencia al consenso en cada interación ${ }^{12}$. Generalmente tres rondas suelen ser suficientes para lograr el consenso, los ajustes más importantes se presentan en la primera y la segunda ronda ${ }^{13}$.

\section{METODOLOGÍA}

De acuerdo con la propuesta de rediseñar y validar el CECOP-23, se presentan a continuación las fases para desarrollar la técnica Delphi.

Se integró un grupo de 7 profesionales de enfermería con experiencia en la práctica clínica, interesados en la comunicación interpersonal de la enfermera y del paciente.

Previa aceptación como integrantes del grupo de expertos, se llevó a cabo un taller de trabajo conjunto con el fin de contextualizar el desarrollo del trabajo programado.

La sesión se organizó en tres momentos, con la participación de las investigadoras responsables. Al inicio se planeó una exposición centrada en la importancia que tiene la comunicación entre la enfermera y el paciente en la oferta de los servicios de salud con énfasis en el comportamiento humanístico, se destacó que, para la mayoría de mediciones de calidad fundamentadas en la dimensión interpersonal, es muy importante la evaluación que hace el paciente desde su percepción la cual incluye el nivel de satisfacción con la atención.

En un segundo momento, se recordó al grupo de expertos el lugar que ocupa un instrumento de investigación en el desarrollo de proyectos con enfoque cuantitativo y el papel que cumple en la recolección de la información de una investigación, su diseño, el constructo que lo fundamenta, las dimensiones y variables que lo integran y sus propiedades de validez y confiabilidad. Se hizo un breve resumen sobre el diseño y validación del CECOP-23, realizados con anterioridad.

Para terminar, se abordaron conceptos necesarios para la operacionalización de los grupos Delphi, el valor de la constitución de consensos de grupo sin que los integrantes se reúnan físicamente, la aplicación de los cuestionarios a distancia, anónimos, secuenciados, con retroalimentación controlada por las coordinadoras. Se destacó la oportunidad que tiene cada integrante del panel de expertos para exponer y evaluar cada propuesta, a fin de garantizar que cada evaluación tenga el mismo valor, se concede la oportunidad de reaccionar a las afirmaciones de los otros participantes y repensar sus evaluaciones ${ }^{11-13}$. Al concluir el taller, se estableció la mecánica de trabajo con base en los lineamientos de la aplicación de la técnica.

Se realizaron tres rondas a distancia, anónimas, con oportunidad de participación de cada experto y con el beneficio de recibir retroalimentación periódica, esta última analizada y organizada por las tres coordinadoras a partir de la información enviada por cada experto. El objeto de análisis correspondió al CECOP-23, mismo que fue enviado en un formato exprofeso, con los reactivos del instrumento que comprenden los comportamientos relacionados con las dos dimensiones del instrumento: empatía y respeto. Cada experto en forma individual analizó su contenido, calificó su idoneidad y pertinencia en una escala de cero a cuatro, además contó con un espacio para 
observaciones en el que sustentó su puntaje, sugirió cambios relacionados con las palabras empleadas, eliminación del reactivo o sustitución de éste. Las coordinadoras analizaron e integraron la información enviada por los expertos con el propósito de preparar el material de las siguientes rondas.

Las coordinadoras realizaron los procedimientos estadísticos para verificar la validez de contenido de cada reactivo (CVR) y la validez integral del instrumento (CVI), apoyándose en los lineamientos de Lawshe ${ }^{14} y$ Tristán ${ }^{15}$. Los valores obtenidos en las tres rondas permitieron determinar que las rondas realizadas eran suficientes.

\section{RESULTADOS}

Con base en los formatos contestados por los profesionales de enfermería expertos, se analizaron los puntajes y las observaciones enviadas por cada uno, se realizaron los procedimientos estadísticos de la primera ronda, se obtuvieron los CVR de cada reactivo y un CVI de 0.84 , mismos que se pueden apreciar en la Tabla 1.

Tabla 1. Resultados de primera ronda del Panel Delphi para la validación del instrumento CECOP-23

\begin{tabular}{|c|c|c|c|c|c|c|c|c|c|}
\hline \multicolumn{10}{|c|}{ Calificación del experto } \\
\hline Reactivo & No. 1 & No. 2 & No. 3 & No. 4 & No. 5 & No. 6 & No. 7 & TOTAL & CVR \\
\hline 1 & 4 & 3 & 4 & 3 & 3 & 3 & 3 & $23 / 28$ & .82 \\
\hline 2 & 4 & 4 & 3 & 3 & 3 & 4 & 3 & $24 / 28$ & .86 \\
\hline 3 & 4 & 4 & 3 & 3 & 3 & 4 & 2 & $23 / 28$ & .82 \\
\hline 4 & 3 & 3 & 3 & 3 & 3 & 3 & 4 & $22 / 28$ & .79 \\
\hline 5 & 3 & 3 & 4 & 3 & 3 & 4 & 2 & $22 / 28$ & .79 \\
\hline 6 & 3 & 4 & 4 & 3 & 3 & 3 & 4 & $24 / 28$ & .86 \\
\hline 7 & 3 & 4 & 4 & 3 & 3 & 3 & 4 & $24 / 28$ & .86 \\
\hline 8 & 4 & 4 & 4 & 3 & 3 & 4 & 4 & $26 / 28$ & .93 \\
\hline 9 & 4 & 3 & 4 & 3 & 3 & 3 & 4 & $24 / 28$ & .86 \\
\hline 10 & 4 & 4 & 3 & 3 & 3 & 3 & 4 & $24 / 28$ & .86 \\
\hline 11 & 4 & 4 & 4 & 3 & 3 & 3 & 4 & $25 / 28$ & .89 \\
\hline 12 & 4 & 4 & 3 & 3 & 3 & 4 & 3 & $24 / 28$ & .86 \\
\hline 13 & 4 & 3 & 4 & 3 & 3 & 3 & - & $20 / 28$ & .71 \\
\hline 14 & 3 & 4 & 3 & 3 & 3 & 4 & 4 & $24 / 28$ & .86 \\
\hline 15 & 4 & 4 & 3 & 3 & 3 & 3 & 4 & $24 / 28$ & .86 \\
\hline 16 & 4 & 3 & 3 & 2 & 3 & 3 & 3 & $21 / 28$ & .75 \\
\hline 17 & 4 & 2 & 3 & 3 & 3 & 3 & 4 & $22 / 28$ & .79 \\
\hline 18 & 4 & 3 & 3 & 3 & 3 & 3 & 3 & $22 / 28$ & .79 \\
\hline 19 & 4 & 4 & 3 & 3 & 3 & 4 & 4 & $25 / 28$ & .89 \\
\hline 20 & 4 & 4 & 3 & 3 & 3 & 3 & 4 & $24 / 28$ & .86 \\
\hline 21 & 4 & 3 & 3 & 4 & 3 & 3 & 4 & $24 / 28$ & .86 \\
\hline 22 & 3 & 4 & 3 & 3 & 3 & 3 & 4 & $23 / 28$ & .82 \\
\hline 23 & 4 & 4 & 4 & - & - & 4 & 4 & $20 / 20$ & 1.00 \\
\hline
\end{tabular}

En la primera ronda, la mayoría de los expertos sugirieron cambios de redacción en todos los reactivos a fin de propiciar una mayor cercanía: hablando de tú; algunos participantes en el afán de ser más claros incrementaron el número de palabras en algún reactivo, aspectos que se analizaron y se retomaron en lo posible. 
En cuanto al primer reactivo: Le sonríen, la mayoría sugirió explorarlo con dos modalidades: me sonrien amablemente cuando se presentan conmigo y me sonrien amablemente cuando me atienden. Los reactivos 5, 16, 18 y 22 fueron eliminados, la mayoría los consideró innecesarios o repetitivos. El reactivo 5 , fue sustituido por la segunda modalidad propuesta para el primer reactivo de la primera ronda, mencionado en este mismo párrafo, con la intención de evitar la inducción de la respuesta, al dejar dos reactivos similares secuenciados. El 16 fue sustituido por otro que se agregó con base en las observaciones de los expertos, con el fin de explorar el respeto a creencias religiosas y a las costumbres que en el CECOP-23 se exploraban de manera más general: respetan su forma de pensar.

Considerando las observaciones emitidas por los expertos, se constituyó el formato para la segunda ronda y se envió a los participantes. Con los valores obtenidos a partir de las respuestas a esta segunda ronda, se procesaron los coeficientes correspondientes a cada reactivo y al instrumento completo, obteniendo un CVI de 0.95 (ver Tabla 2).

Tabla 2. Resultados de segunda ronda del Panel Delphi para la validación del instrumento CECOP-23

\begin{tabular}{|c|c|c|c|c|c|c|c|c|c|}
\hline \multicolumn{10}{|c|}{ Calificación del experto } \\
\hline Reactivo & No. 1 & No. 2 & No. 3 & No. 4 & No. 5 & No. 6 & No. 7 & TOTAL & CVR \\
\hline 1 & 4 & 4 & 3 & 4 & 4 & 3 & 3 & $25 / 28$ & .89 \\
\hline 2 & 4 & 4 & 4 & 3 & 4 & 4 & 3 & $26 / 28$ & .93 \\
\hline 3 & 4 & 4 & 3 & - & 4 & 4 & 3 & $22 / 24$ & .92 \\
\hline 4 & 4 & 4 & 4 & 4 & 4 & 4 & 2 & $26 / 28$ & .93 \\
\hline $5{ }^{*} 1$ bis & 4 & 4 & 1 & 4 & 4 & 3 & 4 & $24 / 28$ & .86 \\
\hline 6 & 4 & 4 & 4 & 4 & 4 & 4 & 3 & $27 / 28$ & .96 \\
\hline 7 & 4 & 4 & 4 & 4 & 4 & 3 & 3 & $26 / 28$ & .93 \\
\hline 8 & 4 & 4 & 4 & 4 & 4 & 4 & 4 & $28 / 28$ & 1.00 \\
\hline 9 & 4 & 4 & 4 & 4 & 3 & 4 & 3 & $26 / 28$ & .93 \\
\hline 10 & 4 & 4 & 4 & 4 & 4 & 4 & 3 & $27 / 28$ & .96 \\
\hline 11 & 4 & 4 & 4 & 4 & 4 & 4 & 3 & $27 / 28$ & .96 \\
\hline 12 & 4 & 4 & 4 & 4 & 4 & 3 & 3 & $26 / 28$ & .93 \\
\hline 13 & 4 & 4 & 4 & 4 & 3 & 4 & 4 & $27 / 28$ & .96 \\
\hline 14 & 4 & 4 & 4 & 4 & 4 & 4 & 4 & $28 / 28$ & 1.00 \\
\hline 15 & 4 & 4 & 4 & 4 & 3 & 4 & 4 & $27 / 28$ & .96 \\
\hline $16^{* *} 17 \mathrm{bis}$ & 4 & 4 & 4 & 4 & 4 & 4 & 4 & $28 / 28$ & 1.00 \\
\hline 17 & 4 & 4 & 4 & 4 & 4 & 4 & 4 & $28 / 28$ & 1.00 \\
\hline 19 & 4 & 4 & 4 & 4 & 4 & 4 & 3 & $27 / 28$ & .96 \\
\hline 20 & 4 & 4 & 4 & 4 & 4 & 3 & 3 & $26 / 28$ & .93 \\
\hline 21 & 4 & 4 & 4 & 4 & 3 & 4 & 4 & $27 / 28$ & .96 \\
\hline 23 & 4 & 4 & 4 & 4 & - & 3 & 4 & $23 / 24$ & .96 \\
\hline
\end{tabular}

* El reactivo 5 original se eliminó y se sustituyó por el 1 bis; ${ }^{* *}$ el reactivo 16 original se eliminó y se sustituyó por el 17 bis; los reactivos 18 y 22 originales se eliminaron. $C V I=551 / 580=.95$

En esta segunda fase los expertos se abocaron a la búsqueda de redacciones más sencillas y cálidas. Se planteó la duda sobre la eliminación del reactivo 7, sin embargo, se dejó para la tercera ronda buscando un mayor consenso, y se integró nuevamente el instrumento para llevar a cabo la tercera ronda.

Los profesionales expertos enviaron los formatos como producto de la tercera ronda, los resultados obtenidos permitieron procesar una vez más los CVR y CVI, éste último fue de 0.96 (ver Tabla 3). 
Tabla 3. Resultados de tercera ronda del Panel Delphi para la validación del instrumento CECOP-23

\begin{tabular}{|c|c|c|c|c|c|c|c|c|c|}
\hline \multicolumn{10}{|c|}{ Calificación del experto } \\
\hline Reactivo & No. 1 & No. 2 & No. 3 & No. 4 & No. 5 & No. 6 & No. 7 & TOTAL & CVR \\
\hline 1 & 4 & 4 & 4 & 3 & 4 & 4 & 3 & $26 / 28$ & .93 \\
\hline 2 & - & 4 & 4 & 3 & 4 & 4 & 3 & $22 / 24$ & .92 \\
\hline 3 & 4 & 4 & 4 & 3 & 4 & 4 & 3 & $26 / 28$ & .93 \\
\hline 4 & 4 & 4 & 4 & 4 & 4 & 4 & 3 & $27 / 28$ & .96 \\
\hline $5^{*} 1 \mathrm{bis}$ & 4 & 4 & 4 & 4 & 4 & 4 & 4 & $28 / 28$ & 1.00 \\
\hline 6 & 4 & 4 & 4 & 4 & 4 & 4 & 4 & $28 / 28$ & 1.00 \\
\hline 7 & 4 & 4 & 4 & 4 & 4 & 4 & 4 & $28 / 28$ & 1.00 \\
\hline 8 & 4 & 4 & 4 & 3 & 4 & 3 & 4 & $26 / 28$ & .93 \\
\hline 9 & 4 & 4 & 4 & 4 & 4 & 4 & 2 & $26 / 28$ & .93 \\
\hline 10 & 4 & 4 & 4 & 4 & 4 & 4 & 4 & $28 / 28$ & 1.00 \\
\hline 11 & 4 & 4 & 4 & 4 & 4 & 4 & 4 & $28 / 28$ & 1.00 \\
\hline 12 & 4 & 4 & 4 & 2 & 4 & 4 & 4 & $26 / 28$ & .93 \\
\hline 13 & 4 & 4 & 4 & 3 & 4 & 4 & 1 & $24 / 28$ & .86 \\
\hline 14 & 4 & 4 & 4 & 4 & 4 & 4 & 4 & $28 / 28$ & 1.00 \\
\hline 15 & 4 & 4 & 4 & 4 & 4 & 4 & 3 & $27 / 28$ & .96 \\
\hline $16^{* *} 17 \mathrm{bis}$ & 4 & 4 & 4 & 3 & 4 & 4 & 4 & $27 / 28$ & .96 \\
\hline 17 & 4 & 4 & 4 & 3 & 4 & 4 & 4 & $27 / 28$ & .96 \\
\hline 19 & 4 & 4 & 4 & 3 & 4 & 4 & 4 & $27 / 28$ & .96 \\
\hline 20 & 4 & 4 & 4 & 4 & 3 & 4 & 4 & $27 / 28$ & .96 \\
\hline 21 & 4 & 4 & 4 & 4 & 4 & 4 & 4 & $28 / 28$ & 1.00 \\
\hline 23 & 4 & 4 & 4 & 4 & 4 & 3 & 4 & $27 / 28$ & .96 \\
\hline
\end{tabular}

${ }^{*}$ El reactivo 5 original se eliminó y se sustituyó por el 1 bis; ${ }^{* *}$ el reactivo 16 original se eliminó y se sustituyó por el 17 bis; los reactivos 18 y 22 originales se eliminaron. CVI $=561 / 584=.96$

En la tercera ronda, la mayoría de los expertos hicieron pocas recomendaciones, enfocadas al cambio de algunas palabras. Uno de los participantes sugirió cambios en los reactivos 8 y 13, los cuales fueron analizados por las coordinadoras, quienes observaron que el cambio implicaba regresar a las propuestas de la primera y segunda rondas, mismas que ya habían sido superadas.

Los valores alcanzados en el CVI de la segunda y tercera ronda, 0.95 y 0.96 respectivamente, sustentaron la decisión de no hacer otra ronda más y considerar al instrumento un producto útil para continuar con la validación de constructo, proceso que será objeto de una nueva etapa de la presente investigación.

El nuevo instrumento CECOP, quedó constituido por 21 reactivos (CECOP-21), diez que exploran empatía y once que exploran respeto (ver anexo 2).

\section{DISCUSIÓN}

El CECOP-21, obtenido como producto del trabajo con los expertos descrito en las páginas anteriores, permite explorar comportamientos de comunicación entre la enfermera y el paciente que respaldan la impartición del cuidado asistencial y terapéutico específico que brindan las enfermeras a las personas hospitalizadas; la selección de profesionales de enfermería que laboran en el campo clínico y que han mostrado interés especial por la comunicación enfermera paciente, reforzado con el desarrollo de un taller para su contextualización en aspectos humanísticos específicos como es el valor de la comunicación interpersonal y su repercusión en los ámbitos hospitalarios, así como la 
Anexo 2. Comportamiento de las Enfermeras en cuanto a su forma de Comunicación Observada por el Paciente (CECOP 21)

\begin{tabular}{|c|c|c|c|c|c|}
\hline LAS ENFERMERAS (OS)... & $\begin{array}{l}\text { Siempre o } \\
\text { casi siempre }\end{array}$ & $\begin{array}{l}\text { Muchas } \\
\text { veces }\end{array}$ & $\begin{array}{l}\text { Algunas } \\
\text { veces }\end{array}$ & $\begin{array}{l}\text { Pocas } \\
\text { veces }\end{array}$ & $\begin{array}{l}\text { Casi nunca } \\
\text { o nunca }\end{array}$ \\
\hline \multicolumn{6}{|l|}{$\begin{array}{l}\text { Sonríen amablemente cuando se presentan } \\
\text { conmigo. E }\end{array}$} \\
\hline \multicolumn{6}{|l|}{$\begin{array}{l}\text { Parece que se molestan cuando no quiero } \\
\text { comer. R }\end{array}$} \\
\hline \multicolumn{6}{|l|}{$\begin{array}{l}\text { Insisten en que me bañe sin escuchar mis } \\
\text { motivos. R }\end{array}$} \\
\hline \multicolumn{6}{|l|}{$\begin{array}{l}\text { Parece que se molestan cuando ensucio la cama } \\
\text { accidentalmente (con comida, agua, pipi o popo). R }\end{array}$} \\
\hline \multicolumn{6}{|l|}{ Son amables cuando me atienden. E } \\
\hline \multicolumn{6}{|l|}{ Se dan su tiempo para hablar conmigo. E } \\
\hline \multicolumn{6}{|l|}{$\begin{array}{l}\text { Me llaman por mi nombre cuando están } \\
\text { conmigo. R }\end{array}$} \\
\hline \multicolumn{6}{|l|}{$\begin{array}{l}\text { Me explican lo que me van a realizar con } \\
\text { palabras que sí entiendo. } R\end{array}$} \\
\hline \multicolumn{6}{|l|}{ Me animan con sus palabras. E } \\
\hline \multicolumn{6}{|l|}{ Se presentan conmigo por su nombre. $\mathrm{R}$} \\
\hline \multicolumn{6}{|l|}{ Atienden oportunamente a mi llamado. $\mathrm{R}$} \\
\hline \multicolumn{6}{|l|}{ Bromean conmigo para darme confianza. E } \\
\hline \multicolumn{6}{|l|}{ Me explican lo que me van a hacer. $\mathrm{R}$} \\
\hline \multicolumn{6}{|l|}{$\begin{array}{l}\text { Me hacen sentir tranquilo cuando están } \\
\text { conmigo. E }\end{array}$} \\
\hline \multicolumn{6}{|l|}{ Platican conmigo mientras me atienden. E } \\
\hline \multicolumn{6}{|l|}{ Respetan mis creencias religiosas. $\mathrm{R}$} \\
\hline \multicolumn{6}{|l|}{ Respetan mis costumbres. $\mathrm{R}$} \\
\hline \multicolumn{6}{|l|}{ Me escuchan aunque estén muy ocupadas. E } \\
\hline \multicolumn{6}{|l|}{ Parece que se molestan cuando me quejo. R } \\
\hline \multicolumn{6}{|l|}{ Me siento “apapachado” por ellas. E } \\
\hline Se interesan por conocer cómo me siento. E & & & & & \\
\hline
\end{tabular}

retroalimentación periódica, con espacios de reflexión individual; favoreció el análisis que hicieron de cada comportamiento, sugiriendo palabras y formas de expresión más cercanas a la realidad de sus pacientes.

El instrumento Caring Assessment Tool (CAT) desarrollado por Duffy ${ }^{16,17}$ en cuanto a la comunicación enfermera paciente, ha tenido una larga evolución en su desarrollo y ha sido objeto de diversas fases de validación por expertos y de constructo. En sus primeras etapas refirió que estaba integrado por ocho factores o componentes del cuidado, de los cuales seis coincidían con las dimensiones o factores que integran el CECOP-235 , la última versión del CAT tiene 26 reactivos, Duffy concluyó que el instrumento tiene un solo factor y es la interacción personal. El CECOP-23, antecedente del CECOP-21, contaba con dos dimensiones con un equilibrio en sus reactivos; sin embargo, al someterlo al proceso de validación de constructo perdió esta proporción, concediendo más importancia a la dimensión de comprensión empática o empatía a expensas de la reducción de los reactivos relacionados con el respeto. Conforme a lo antes mencionado, se propone la validación de constructo del CECOP-21 que permitirá explorar si se logra un equilibrio entre las dos dimensiones o factores, o se tendría que hablar de un solo factor difícil de descomponer como concluyó Duffy. 
El hecho de involucrar a expertos en la integración del panel ofrece mayor fiabilidad en esta fase del proceso $0^{18}$, la aplicación de varias rondas estimula la reflexión y confrontación con la propia experiencia ${ }^{13}$, el anonimato tanto en la aplicación como en la retroalimentación de los cuestionarios en cada etapa es importante, ya que proporciona igual oportunidad a cada experto para exponer y evaluar cada idea ${ }^{11}$, el uso de técnicas estadísticas en el procesamiento de los datos reduce la subjetividad de los coordinadores ${ }^{14,15} \mathrm{y}$ la aplicación en el ámbito de salud ha mostrado buenos resultados ${ }^{13}$.

La aplicación de la técnica Delphi permite $\operatorname{afirmar}^{8}$ que el juicio de los expertos es un procedimiento fundamental en cuanto a la validez de contenido y a la pertinencia del uso de los reactivos de un instrumento, puesto que la validez de consenso derivada de rondas con retroalimentación periódica conduce a acuerdos sobre los contenidos explorados.

Leal et al. ${ }^{8}$ al abordar el comportamiento empatía, encontraron que los expertos indistintamente clasificaban los reactivos como actitud empática o como escucha activa por la dificultad conceptual para separarlas, ya que la actitud empática englobaría a la escucha activa y a la respuesta empática. Cabe señalar que en el análisis del CECOP-21 no se exploró esta diferencia conceptual, pero la comprensión empática claramente contiene la escucha activa como puede apreciarse en los reactivos 18 Me escuchan, aunque estén muy ocupadas y 21 Se interesan por conocer cómo me siento; reactivos que corresponden a la dimensión de empatía.

Morales et al. ${ }^{10}$ en un ejercicio de validación y estandarización de un instrumento de evaluación de los comportamientos, también hicieron uso del panel de expertos y aunque parten de propósitos diferentes, se coincide en la libertad que debe tener cada experto para hacer adecuaciones a la redacción de los reactivos, con el fin de que el comportamiento guarde congruencia con el concepto evaluado y se adecúe a la realidad mexicana. En el caso del CECOP-21, es claro que el reactivo $20 \mathrm{Me}$ siento apapachado por ellas, responde a un concepto derivado de la lengua náhuatl y muy propio de nuestro país.

Parrado et al. ${ }^{19}$ con el apoyo de las discusiones de las observaciones de los expertos, llegaron al consenso de sus reactivos definitivos para la validación de dos instrumentos que miden la relación interpersonal de la enfermera con el paciente y su familia en una unidad de cuidado intensivo, en el caso del CECOP-21 la retroalimentación anónima entre expertos también permitió la integración de este instrumento.

Poblete et al..$^{20}$,igualmente se apoyaron en grupo de expertos para la validación de su instrumento, no obstante; su propósito se dirigió a la opinión de éstos en cuanto al sustento del instrumento en la teoría del cuidado humano, cuestionamiento que no se abordó en el caso del CECOP-21, dado que este instrumento ya tiene antecedentes en cuanto a las dimensiones que lo integran.

Estudios realizados en España ${ }^{21} y$ en Colombia ${ }^{22}$ comparten el reconocimiento de trabajar con expertos y la técnica Delphi para favorecer la validación de contenido de instrumentos relacionados con la comunicación en el ámbito de enfermería, estrategia aplicada en el CECOP-21. De igual forma, Bonilla ${ }^{23}$ y Achury, ${ }^{24}$ aunque exploran otras áreas no dirigidas a la comunicación, refieren la importancia del uso del panel Delphi y la aplicación de coeficientes validez de contenido CVR y CVI, empleada en desarrollo de este reporte.

\section{CONCLUSIONES}

La validación de contenido, propósito de este artículo, nos permite rescatar reactivos relacionados con la dimensión de respeto que fueron eliminados cuando se realizó la validación de constructo del CECOP-23. 
El trabajo con los expertos ayudó a retomar reactivos del CECOP-23 relacionados con las dimensiones de empatía y respeto, pero redactados de forma más cálida y profunda. Lo anterior permitió ahondar en algunos aspectos que contribuyen a una mejor exploración del constructo comunicación enfermera paciente, que por su complejidad es difícil de fragmentar, como lo reportan algunos autores.

La incorporación de enfermeras con experiencia en la práctica y sensibles a las relaciones interpersonales enfermera-paciente, favorecieron la reestructuración con reactivos afectuosos enmarcados en un contexto local y con más potencial de medición. El diseño y aplicación de este tipo de herramientas pueden ser utilizados en las instituciones de salud como parte de la dimensión interpersonal de la calidad.

\section{RESPONSABILIDADES ÉTICAS}

Protección de personas y animales. Los autores declaran que para esta investigación no se han realizado experimentos en seres humanos y animales.

Confidencialidad de los datos. Los autores declaran que no se brindan datos que permitan identificar a los participantes.

Derecho a la privacidad y consentimiento informado. Los autores han obtenido el consentimiento informado de los participantes.

Financiamiento. Ninguno.

Conflicto de intereses. Ninguno.

\section{REFERENCIAS}

1. Dirección General de Calidad y Educación en Salud/ Comisión Interinstitucional de Enfermería. Evaluación de la calidad de los Servicios de Enfermería. Tres indicadores de aplicación hospitalaria. México: Secretaria de Salud; 2003. http://bit.ly/2miHrL

2. Tay LH, Hegney D. Factors affecting effective communication between registered nurses and cancer patients in an inpatient setting: a systematic review. Int J Evid Based Healthc. 2011; 9(2): 151-64 https://doi.org/10.1111/j.1744-1609.2011.00212.x

3. Olano-Lizárraga M, García-Vivar C. Visión de los pacientes sobre el cuidado de enfermería. Una revisión de la literatura. Rev Rol Enferm. 2012; 85(1): 20-31.

4. Müggenburg C, Riveros-Rosas A, Juárez-García F. Entrenamiento en habilidades de comunicación en enfermeras y percepción del paciente que recibe su cuidado. Enferm. univ. 2016;13(4): 201-207. http://dx.doi.org/10.1016/j.reu.2016.08.001

5. Müggenburg C, Robles R, Valencia A, Hernández-Guillén MC, Olvera S, Riveros Rosas A. Evaluación de la percepción de pacientes sobre el comportamiento de comunicación del personal de enfermería: diseño y validación en población mexicana. Salud Ment. 2015; 38(4): 273-80.

http://dx.doi.org/10.17711/SM.0185-3325.2015.037

6. Cormier WH, Cormier LS. Estrategias de entrevista para terapeutas: habilidades básicas e intervenciones cognitivo-conductuales. $3^{\text {a }}$. ed. Bilbao: Declée de Brouwer; 2000.

7. Guijón-Alvarado MA, Müggenburg-Rodríguez Vigil C. Instrumentos que miden la comunicación enfermera paciente. Enferm. univ. 2019;16(3):303-12.https://doi.org/10.22201/eneo.23958421e.2019.3.687

8. Leal-Costa C, Tirado-González S, van-der Hofstadt-Román CJ, Rodríguez-Marín J. Creación de la Escala sobre Habilidades de comunicación en Profesionales de la Salud, EHC-PS. Anal Psicol. 2016; 32(1): 49-59. http://dx.doi.org/10.6018/analesps.31.3.184701 
9. Parrado-Lozano YM, Sáenz-Montoya X, Soto-Lesmes VI, Guáqueta-Parada SR, Amaya-Rey P, et al. Validez de dos instrumentos para medir la relación interpersonal de la enfermera con el paciente y su familia en la unidad de cuidado intensivo. Investig. enferm. imagen desarro. 2015; 18(1):115-28. https://doi.org/10.11144/Javeriana.ie18-1.vimr

10. Morales-Castillo FA, Hernández-Cruz MC, Morales MCR, Landeros EAO. Validación y estandarización del instrumento: Evaluación de los comportamientos de cuidado otorgado en enfermeras mexicanas. Enferm. univ. 2016; 13(1): 3-11. http://dx.doi.org/10.1016/j.reu.2015.11.005

11. Yañez-Gallardo R, Cuadra-Olmos R. La Técnica Delphi y la investigación en los servicios de salud. Cienc. Enferm. 2008; 16(1):9-15. http://dx.doi.org/10.4067/So717-95532008000100002

12. Powell C. The Delphi technique: myths and realities. J Adv Nurs. 2003; 41(4):376-82. https://doi.org/10.1046/j.1365-2648.2003.02537.x

13. De Villiers MR, De Villiers PJ, Kent AP. The Delphi technique in health sciences education research. Med Teach. 2005; 27(7): 639-43. https://doi.org/10.1080/13611260500069947

14. Lawshe CH. A quantitative approach to content validity. Pers. Psychol. 1975; 28(4): 563-75. http://dx.doi.org/10.1111/j.1744-6570.1975.tbo1393.x

15. Tristán-López A. Modificación al Modelo de Lawshe para el dictamen cuantitativo de la validez de contenido de un instrumento objetivo. Avances en Medición. 2008; 6(1): 37-48. http://bit.ly/2lZyuaw

16. Duffy JR, Hoskins L, Seifert RF. Dimensions of caring: psychometric evaluation of the caring assessment tool. Adv Nurs Sci.2007; 30(3): 235-45. https://doi.org/10.1097/01.ANS.0000286622.84763.a9

17. Duffy J, Brewer B, Weaver M. Revision and psychometric properties of the Caring Assessment Tool. Clin Nurs Res. 2010; 23(1): 80-93. https://doi.org/10.1177/1054773810369827

18. Okoli C, Pawlowski S. The Delphi method as a research tool: An example, design, considerations and applications. Inform manage-Amster. 2004; 42 (1): 15-29. https://doi.org/10.1016/j.im.2003.11.002

19. Newman AR, Helft PR. Reliability and Validity of a Tool to Assess Oncology Nurses Experiences With Prognosis-Related Communication. Oncol Nurs Forum. 2015; 42(1): 64-73. https://doi.org/10.1188/15. ONF.64-73

20. Poblete-Troncoso MC, Valenzuela-Suazo SV, Merino JM. Validación de dos escalas utilizadas en la medición del cuidado humano transpersonal basadas en la Teoría de Jean Watson. Aquichán. 2012; 12(1): 8-21. http://bit.ly/2kg1igo

21. Morales-Asencio JM, Bonill-De las Nieves C, Celdrán-Mañas M, Morilla-Herrera JC, Martín-Santos FJ, Contreras-Fernández E, et al. Diseño y validación de instrumento de evaluación de la satisfacción con los servicios de atención domiciliaria: SATISFAD. Gac Sanit. 2007; 21(2):106-13.http://bit.ly/2kmMiM5

22. Inés-Parra D, Arango-Bayer GL. Validez e confiabilidade das escalas de comunicação e coordenação para mensurar o papel interdependente da enfermagem. Av. Enferm. 2010; 28(1): 51-62. http://bit.ly/2kk81El

23. Bonilla-Ibañez CP, Gutiérrez de Reales E. Desarrollo y características psicométricas del instrumento para evaluar los factores que influyen en la adherencia a tratamientos farmacológicos y no farmacológicos en personas con factores de riesgo cardiovascular. av. enferm. 2014; 32(1): 53-62. http://dx.doi.org/10.15446/av.enferm.v32n1.46035

24. Achury-Beltrán LF. Validez y confiabilidad del cuestionario para medir la adherencia al tratamiento de pacientes con enfermedad cardiovascular. Aquichan. 2017; 17(4): 460-71. http://dx.doi.org/10.5294/aqui.2017.17.4.9 